University of Warwick institutional repository: http://go.warwick.ac.uk/wrap This paper is made available online in accordance with publisher policies. Please scroll down to view the document itself. Please refer to the repository record for this item and our policy information available from the repository home page for further information.

To see the final version of this paper please visit the publisher's website. access to the published version may require a subscription.

\author{
Author(s): Swaran P Singh \\ Article Title: Review: Black and minority ethnic people are more likely to \\ be detained under the Mental Health Act 1983-no clear evidence why \\ Year of publication: 2008 \\ Link to published version: http://dx.doi.org/doi:10.1136/ebmh.11.2.61 \\ Publisher statement: None
}




\title{
Review: Black and minority ethnic people are more likely to be detained under the Mental Health Act 1983 - no clear evidence why
}

\author{
Swaran P Singh
}

Correspondence to: Swaran P Singh, Health Sciences Research Institute, Warwick Medical School, Coventry CV4 7AL, UK; S.P.Singh@warwick.ac.uk

\section{QUESTION}

\section{Question:}

Compared with White people, how frequently are people from Black and Minority Ethnic groups detained under the Mental Health Act 1983?

\section{Outcomes:}

Rates of compulsory detention under the Mental Health Act.

\section{METHODS}

Design: Systematic review with meta-analysis.

Data sources: MEDLINE, EMBASE, CINAHL, PsycINFO, ASSIA, SIGLE, HMIC, Web of Science, Cochrane database and the National Research Register; 1984 to April 2005. CD-ROM for the British National Bibliography was also searched for relevant literature.

Study selection and analysis: English language studies relating to compulsory detention under the Mental Health Act 1983 and including terms related to mental illness or forensic psychiatry, and with inclusion of 2 ethnic groups (any non-White). Odds of compulsory detention of people from Black and Minority Ethnic (BME) groups compared with White groups were combined using a fixed effects metaanalysis. Meta-regression was then used to explore reasons for heterogeneity between studies. It was based on subgroup analyses according to episode (first, second, etc), patient type (for example, civil or forensic), study quality, and year of publication. The authors then narratively discuss the possible theories for the difference in detention rates.

\section{MAIN RESULTS}

Forty nine studies met the inclusion criteria and 19 were included in the metaanalysis. Most were cross-sectional studies with 53\% including $<120$ patients; $71 \%$ of studies were conducted in London. BME groups were more likely to be detained under the mental health act than Whites (OR 3.35, 95\% CI 3.05 to 3.73; $\mathrm{p}<0.0001$ ). Separate analysis by ethnic group found that Blacks were detained more frequently than Whites or Asians (OR for Black vs White: 3.83, 95\% CI 3.42 to 4.29; OR Black vs Asian: 2.25, 95\% CI 1.72 to 2.94). By detention type, more BME groups than White were detained for non-forensic reasons (OR 4.03, 95\% CI 3.37 to 4.81; $\mathrm{p}<0.0001$ ) than for forensic reasons (OR 2.29, 95\% CI 1.50 to 3.50; $<<0.0001$ ), and more BME people with first episode illness were detained compared to Whites. Higher quality studies showed less of a difference between Black and White detention 
rates, although there was no effect of study quality on comparisons of total BME population with Whites. Similarly, the difference in risk decreased with more recent publication dates (and there was a link between recent publication date and study quality). In 31\% of studies, racial labelling, discrimination and stereotyping were the most frequently discussed reasons for greater detention rates among BME groups; $26 \%$ of studies discussed dissatisfaction and negative perceptions of psychiatric services; and 22\% each to higher rates of psychosis amongst BME groups, and to greater perception of violence. The researchers conclude that there is no primary evidence to confirm the effects of any of these factors on the difference in detention rates.

\section{CONCLUSIONS}

There is an excess in rates of psychiatric detention of Black Minority Ethnic people compared with Whites. However there is no reliable explanation for this difference.

\section{ABSTRACTED FROM}

Singh SP, Greenwood N, White S, et al. Ethnicity and the Mental Health Act 1983: systematic review. Br J Psychiatry 2007;191:99-105.

\section{FOOTNOTES}

Notes: The underlying studies used in the meta-analysis were of questionable quality. Researchers report that "few studies were hypothesis-driven and only 39\% stated inclusion and exclusion criteria". Meta-regression revealed a significant effect of study quality on the differences in risk between ethnic groups.

Source of funding: Department of Health.

\section{Commentary}

\section{Elizabeth Cantor-Graae}

Department of Clinical Sciences, Lund University, Malmö, Sweden

\section{Commentary Prevalence}

Excessive rates of psychiatric detentions among Black and Minority Ethnic (BME) groups in the UK raise important questions concerning equitable treatment. Previous findings indicate a fourfold increase in rates of compulsory admissions in Black patients compared to White patients. ${ }^{1}$ A variety of hypotheses have been offered for excessive detention rates in BME patients. These include higher rates of psychosis, delayed help-seeking due to social isolation and/or lack of community involvement, and increased referral by police and court authorities. A more controversial hypothesis attributes increased detention rates in BMEs to racial stereotyping and discrimination within psychiatry. ${ }^{2}$ The current review and meta-analysis by Singh and colleagues 
represents a much-needed systematic examination of all UK literature on ethnicity and detentions, with particular focus on the strength of the evidence in support of the various hypotheses - that is, patient-related, service-related, culture-related, or patientservice interaction. An innovative feature of the current review is the categorisation of the level of supportive evidence (that is, primary, secondary, no evidence) provided by each individual study. Singh and colleagues' approach to the data is appropriately cautious, and takes into consideration the sensitive nature of the topic.

A finding of particular relevance for clinicians is the increase in detention rates over time, suggesting that the BME patient's relationship with mental health services may become increasingly problematic with successive admissions. However, the review finds little primary support for dissatisfaction or mistrust of mental health services by BME patients or for racism and racial stereotyping of BME patients. Thus, explanations that invoke institutionalised racism in psychiatry as the underlying mechanism contributing to ethnic patterns in compulsory detentions seem to be lacking any firm empirical basis. Although clinicians should be aware of the processes that influence detention decisions, the complex factors that contribute to excess psychiatric detention rates in BMEs may well lie outside the healthcare system.

\section{REFERENCES}

1. Bhui K, Stansfeld S, Hull S, et al. Ethnic variations in pathways to and use of specialist mental health services in the UK. Br J Psychiatry 2003; 182: 10516.[Abstract/Free Full Text]

2. Littlewood R, Lipsedge M. Aliens and alienists: ethnic minorities and psychiatry. 3rd ed. London: Routledge, 1997. 